\title{
Contribution of magnetic resonance imaging to the prenatal diagnosis of common congenital vascular anomalies
}

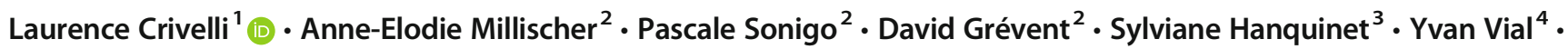 \\ Leonor Alamo ${ }^{1}$
}

Received: 11 May 2020 / Revised: 8 September 2020 / Accepted: 21 February 2021 / Published online: 23 April 2021

(C) The Author(s) 2021

\begin{abstract}
Background Screening ultrasound (US) has increased the detection of congenital vascular anomalies in utero. Complementary magnetic resonance imaging (MRI) may improve the diagnosis, but its real utility is still not well established.

Objectives We aimed to describe the imaging findings on prenatal US and MRI of the most frequent congenital vascular anomalies (lymphatic malformations and congenital hemangiomas) to assess the accuracy of prenatal US and MRI exams for diagnosis and to evaluate the relevance of the additional information obtained by complementary fetal MRI.

Materials and methods All confirmed postnatal congenital vascular anomalies detected in the last 10 years at 3 university hospitals were retrospectively identified. The prenatal diagnosis was compared with the final diagnosis for both methods and the clinical relevance of additional MRI information was evaluated. A second MRI in advanced pregnancy was performed in fetuses with lesions in a sensitive anatomical location and the clinical relevance of the additional information was evaluated.

Results Twenty-four cases were included in the study, 20 lymphatic malformations and 4 hemangiomas. MRI slightly improved the diagnosis of lymphatic malformation, $85 \%$ vs. $80 \%$ at US, especially for abdominal lesions. Both methods had a low identification rate (25\%) for tumors. MRI performed late in five fetuses with lymphatic malformation allowed optimized management at birth.

Conclusion MRI improves the diagnosis of congenital lymphatic malformations whereas hemangiomas remain difficult to identify in utero. The main role of MRI is to provide high-defined anatomical data to guide management at birth.
\end{abstract}

Keywords Congenital hemangiomas · Fetus $\cdot$ Lymphatic malformations $\cdot$ Magnetic resonance imaging $\cdot$ Prenatal diagnosis Ultrasound · Vascular anomalies

Laurence Crivelli

laurence.crivelli@chuv.ch

1 Department of Diagnostic and Interventional Radiology, CHUV - Centre Hospitalier Universitaire Vaudois, Rue du Bugnon 21, 1011 Lausanne, Switzerland

2 Department of Pediatric Radiology, Hôpital Universitaire Necker - Enfants Malades, Paris, France

3 Department of Radiology, HUG - Hôpitaux Universitaires de Genève, Genève, Switzerland

4 Department of Gynecology and Obstetrics, CHUV - Centre Hospitalier Universitaire Vaudois, Lausanne, Switzerland

\section{Introduction}

After decades of confusing nomenclature, the International Society for the Study of Vascular Anomalies (ISSVA) adopted in 1996 the classification suggested by Mullicken and Glowacki in 1982 [1], which distinguishes between:

(1) Vascular malformations, which are developmental alterations of vascular channels. They are classified according to the predominant type of vessel in the malformation into arterial, venous, capillary, lymphatic or mixed [2, $3]$, and have a characteristic high- or low-flow pattern in the presence or absence of an arterial component [1,2]. They may be part of an "overgrowth disorder" in a wide variety of 
syndromes that combine a vascular malformations with a dysplastic, enlarged bone, limb or even a body-half $[3,4]$.

(2) Vascular tumors, which are formed by proliferative and hyperplastic endothelial lesions and typically have a highflow arterial pattern. In contrast to the most common vascular tumor, benign infantile hemangioma, congenital hemangiomas are fully developed at birth. For a long time, vascular tumors were classified according to their postnatal clinical evolution into rapidly involuting congenital hemangiomas (RICH), which have a spontaneous and complete resolution without therapy in $<14$ months, and non-involuting congenital hemangiomas $(\mathrm{NICH})$, which grow with the child, show no regression and may require therapy $[2,4,5]$. More recently, partially involuting hemangiomas (PICH) have been described as an intermediate type that initially behaves as a RICH lasting as a NICH [6].

The generalization of screening ultrasound (US) during pregnancy has increased the rate of detection of vascular anomalies in utero [7, 8]. An accurate prenatal diagnosis may help the medical team evaluate the extension and precise anatomical location of these lesions and improve the information provided to the parents, including predictions regarding prognosis during pregnancy, at birth and in the neonatal period $[2,9]$.

Prenatal magnetic resonance imaging (MRI) can be a complementary imaging method for fetal pathology, but its usefulness in patients with congenital vascular anomalies is not well known. The aims of this study are to describe the prenatal imaging findings on US and MRI of the two most frequently detected congenital vascular anomalies: lymphatic malformations and congenital hemangiomas. We compare the accuracy of both methods for diagnosis and evaluate the relevance of the additional information provided by complementary prenatal MRI for management.

\section{Materials and methods}

\section{Inclusion criteria}

A retrospective chart review of a 10-year period (January 2010 to December 2019) was conducted for all neonates with a confirmed congenital lymphatic malformation or hemangioma with available prenatal US and MRI exams.

The study was performed in three different hospitals, the University Hospitals of Lausanne (CHUV) and Genève (HUG) in Switzerland and the Necker Children's Hospital in Paris (France), and was approved by the three institutional review boards and ethics committees. The clinical data of the patients included in the study were obtained from the Soarian program (Cerner, London, United Kingdom) at the Swiss
hospitals/Mediweb program (Mediweb Solutions, Strasbourg, France) in France and the prenatal imaging exams (US and MRI) from the Archimède program (Archimède Solutions, Geneva, Switzerland) in Switzerland/Astraia program (Astraia Software gmbh, Ismaning, Germany) in France.

\section{Classification of congenital vascular anomalies}

Vascular anomalies were classified according to Mullicken and Glowacki's classification accepted by the ISSVA [1] (Table 1).

\section{Prenatal imaging studies}

Screening US exams, including color Doppler studies, were usually performed between the $19^{\text {th }}$ and $24^{\text {th }}$ week of pregnancy by obstetricians with a wide experience in prenatal diagnosis with the following US systems: Voluson 730/E8/E10 Expert (GE Healthcare, Strasbourg, France) in Switzerland and France and/or Acuson Sequoia (Siemens Healthineers, Erlangen, Germany) in Switzerland.

Complementary MRI exams were performed using a phased-array multi-channel body coil on a 1.5-tesla (T) MR system with the machines and protocols described in Table 2 . MRI exams were evaluated by experienced pediatric radiologists (L.A., P.S., A.-E.M., D.G. and S.H., all with more than 20 years of experience in radiology and more than 15 years of experience in pediatric radiology) who had access to the previous US images and reports.

Standard prenatal imaging reports included descriptions of the size, volume, anatomical location and organ of origin of the detected pathology. The morphology of the lesion mostly solid or cystic, micro- or macrocystic, homogeneous or heterogeneous - its characteristics of echogenicity/signal intensity and its effects on the adjacent organs when performed were also included in the reports.

\section{Review of prenatal studies}

The reports of the prenatal imaging studies and their prospectively suggested diagnoses were obtained from the Radoffice (Medspazio, Geneva, Switzerland) and Soarian programs at the CHUV and Geneva and from Carestream (Philips, Horgen, Switzerland) at Necker. They were compared with the final diagnosis resulting from autopsy, anatomopathological exams, surgical reports, and/or postnatal clinical and imaging findings.

The rate of agreement between the prospectively suggested and final diagnoses for both US and MRI were established and the sensitivity for both methods compared. Finally, the clinical relevance of the additional information obtained at MRI and its consequences on management decisions were registered. 
Table 1 International Society for the Study of Vascular Anomalies classification of congenital vascular pathologies (adapted from [10])

\begin{tabular}{lll}
\hline Tumors & Malformations & \\
\cline { 2 - 3 } & Simple & Combined malformations \\
\hline RICH & Capillary malformations & Capillary-venous \\
& & Capillary-lymphatic \\
NICH & Lymphatic malformations & Lymphatic-venous \\
& & Capillary-lymphatic-venous \\
PICH & Venous malformations & Capillary-arterial-venous \\
& Arteriovenous malformations & Capillary-lymphatic-arterial-venous \\
\hline
\end{tabular}

$\mathrm{NICH}$ non-involuting congenital hemangioma, $\mathrm{PICH}$ partially involuting hemangioma, $\mathrm{RICH}$ rapidly involuting congenital hemangioma

\section{Additional value of MRI studies performed late in pregnancy}

In some patients with lymphatic malformations, a second prenatal MRI was performed close to the due date to optimize management upon delivery, with identical protocol to that previously described. The changes in management secondary to the information provided in these late exams were registered and evaluated.

\section{Results}

The retrospective search identified 24 patients who fit the inclusion criteria, with 20 lymphatic malformations and 4 hemangiomas. The mortality rate was $16.7 \%$ (4/24 patients). Pregnancy was legally terminated in two fetuses with large lymphatic malformations and two neonates died at birth, one because of an extensive thoracic lymphatic malformation with bilateral pleural effusions and lung infiltration and one because of a rapidly growing hepatic hemangioma causing severe pulmonary hypoplasia.

Data concerning the gender of the fetuses, the anatomical location of the lesions, the main imaging findings, the prenatal suggested diagnosis, the final confirmed diagnosis and the outcome of the patients are described in Table 3 for lymphatic malformations and in Table 4 for congenital hemangiomas. These tables also show the correlation between the prenatal US and MRI and between the prenatal and the final diagnoses.

\section{Congenital lymphatic malformations}

Lymphatic malformation was suggested in the prenatal imaging of 17 patients and confirmed after birth for the last 3 cases (Table 3). Most of the lesions (14 cases; 70\%) were located in the face and neck with occasional extension into the axilla and/or the thorax. The remaining six lesions were located at the axilla (one case), the thorax (two cases), or the abdomen and pelvis (three cases). Prenatal US achieved a correct diagnosis in 16 cases

Table 2 Magnetic resonance systems and protocols of fetal magnetic resonance imaging used in the three university hospitals

\begin{tabular}{|c|c|c|c|}
\hline & Brand & Model & Sequences \\
\hline Lausanne, Switzerland & $\begin{array}{l}\text { Siemens Healthineers (Erlangen, } \\
\text { Germany) }\end{array}$ & $\begin{array}{l}\text { Magnetom Symphony } \\
\text { Aera }\end{array}$ & $\begin{array}{l}\text { In the } 3 \text { fetal planes: } \\
\text { T2 half-Fourier acquisition single-shot turbo spin echo } \\
\text { (HASTE) } \\
\text { T2 true fast imaging with steady-state precession (FISP) } \\
\text { T1-weighted volumetric interpolated breath-hold } \\
\text { examination }\end{array}$ \\
\hline Geneva, Switzerland & Siemens Healthineers & Avanto & $\begin{array}{l}\text { T2 HASTE coronal } \\
\text { T2 true FISP sagittal } \\
\text { 2-dimensional T1-weighted spoiled incoherent gradient } \\
\quad \text { echo sequence axial }\end{array}$ \\
\hline Paris, France & GE Healthcare (Waukesha, WI) & Optima MR450w & $\begin{array}{l}\text { In the } 3 \text { fetal planes: } \\
\text { Fast imaging employing steady-state acquisition (FIESTA) } \\
\text { Single-shot fast spin echo } \\
\text { 3-dimensional spoiled gradient echo pulse sequence }\end{array}$ \\
\hline
\end{tabular}




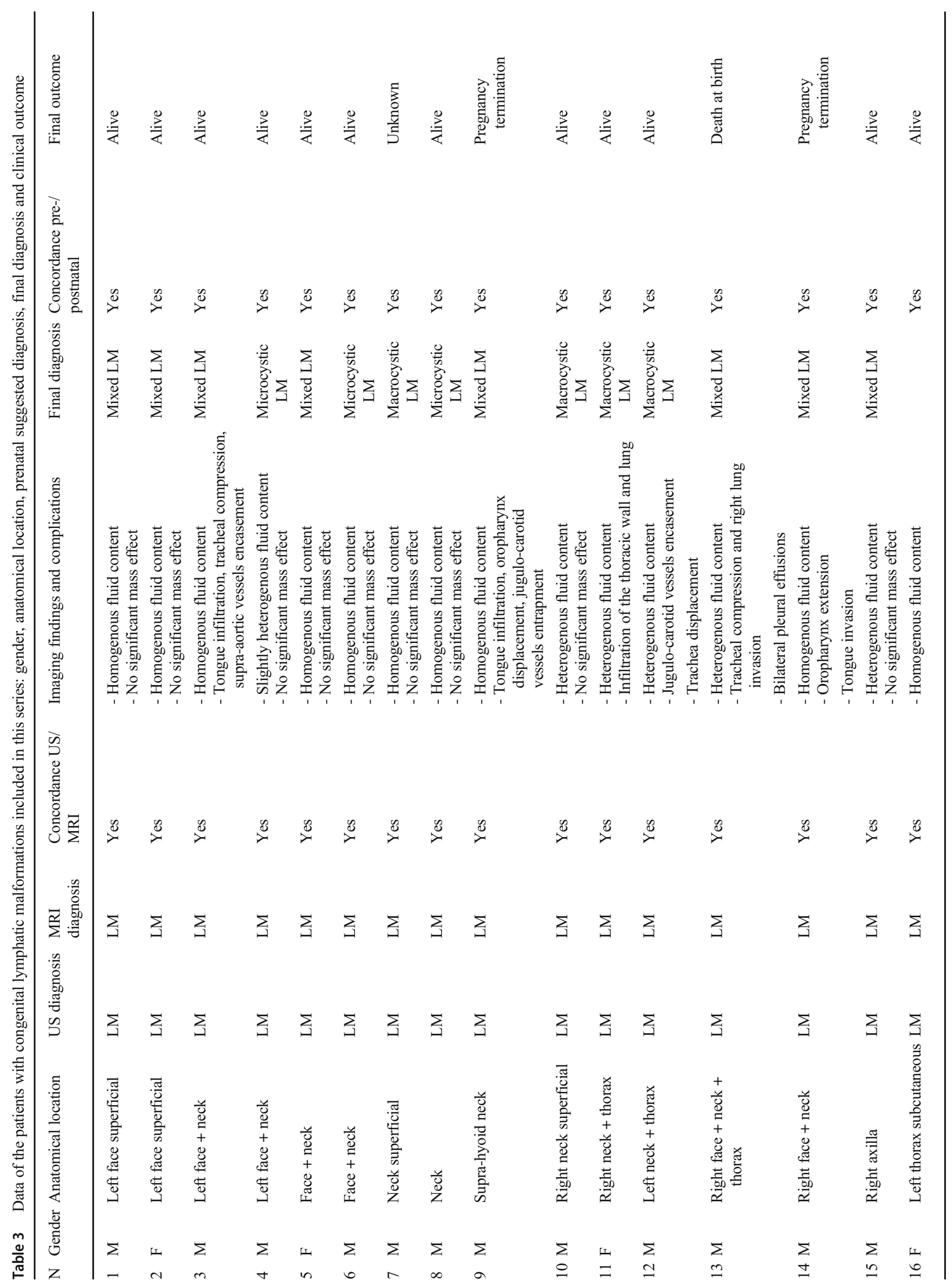




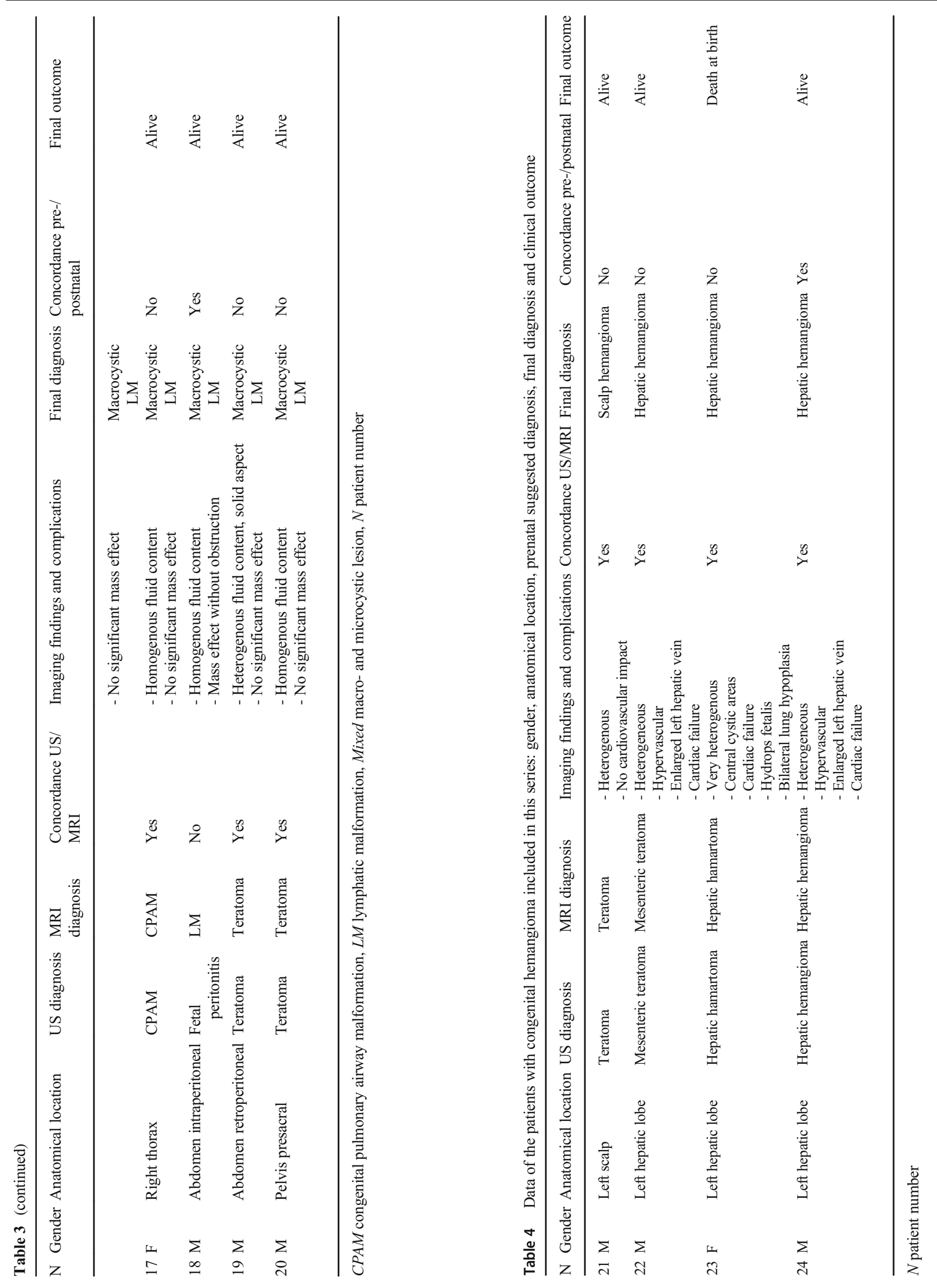


Fig. 1 A male fetus at 26 weeks' gestation with presacral lymphatic malformation (N20, Table 3). a-c Coronal oblique (a), sagittal paramedian (b) and axial (c) T2-weighted MR images (repetition time/echo time 1,200/ $90 \mathrm{~ms}$ ) show the presacral fluid isointense lesion (arrows) with an internal septum. The prenatally suggested diagnosis was a presacral teratoma, based not only on imaging findings but also the typical anatomical location of this common congenital tumor.

Pathology after postnatal surgery identified a macrocystic lymphatic malformation
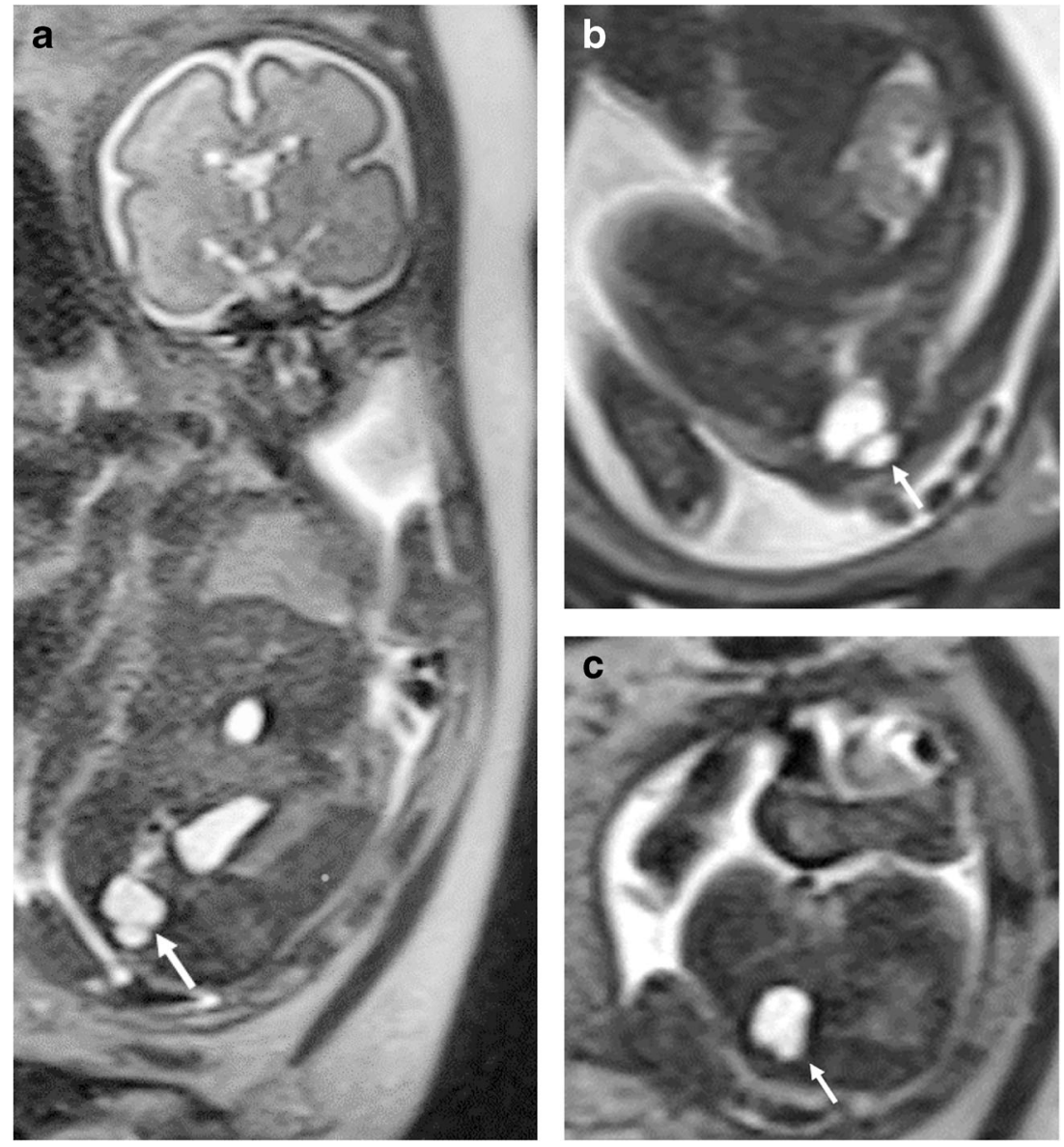

(80\%) with the following anatomical locations: cervical and/or facial (11 cases), axillary (1 case), cervicothoracic (3 cases) or thoracic (1 case). Prenatal MRI identified 17 cases (85\%), the 16 first previously described at US and 1 additional abdominal lesion. In three fetuses with a postnatally confirmed lymphatic malformation, prenatal US and MRI suggested a teratoma for two abdominal lesions (Fig. 1) and a congenital pulmonary airway malformation (CPAM) for a thoracic lesion (Table 3). The only discrepancy between the suggested diagnosis on US and MRI was an extensive mesenteric lymphatic malformation, which was correctly diagnosed on MRI whereas US suggested a peritonitis after intestinal perforation (Fig. 2).

\section{Additional value of MRI studies performed late in pregnancy}

In five fetuses with prenatally diagnosed lymphatic malformations, a second MRI exam was performed during advanced pregnancy (33-36 weeks). Indication for these late studies were an anatomical location close to the upper respiratory airways (five cases) and/or a voluminous lesion (two cases) (Fig. 3). These exams were performed for management at birth, including decisions about the type of delivery and the need for extracorporeal intrapartum treatment (EXIT procedure) [11]. Table 5 includes the additional information provided by these late performed MRIs and its influence on patient management.

\section{Congenital hemangiomas}

The anatomical location of the four confirmed congenital hemangiomas included in this series were the scalp (one case) and the liver (three cases) (Figs. 4 and 5). The prenatal accuracy of diagnosis was very low and showed no difference between the two imaging methods, with only 1 of 4 (25\%) confirmed hemangiomas correctly identified in utero (Table 4).

\section{Discussion}

The ISSVA acceptance of Mullicken and Glowacki's classification for congenital vascular anomalies [1] has contributed considerably to a better understanding of these pathologies while the generalization of screening US has increased their in utero detection rate. Impressive technical advances and an increased availability of MRI have led to an extension of this 

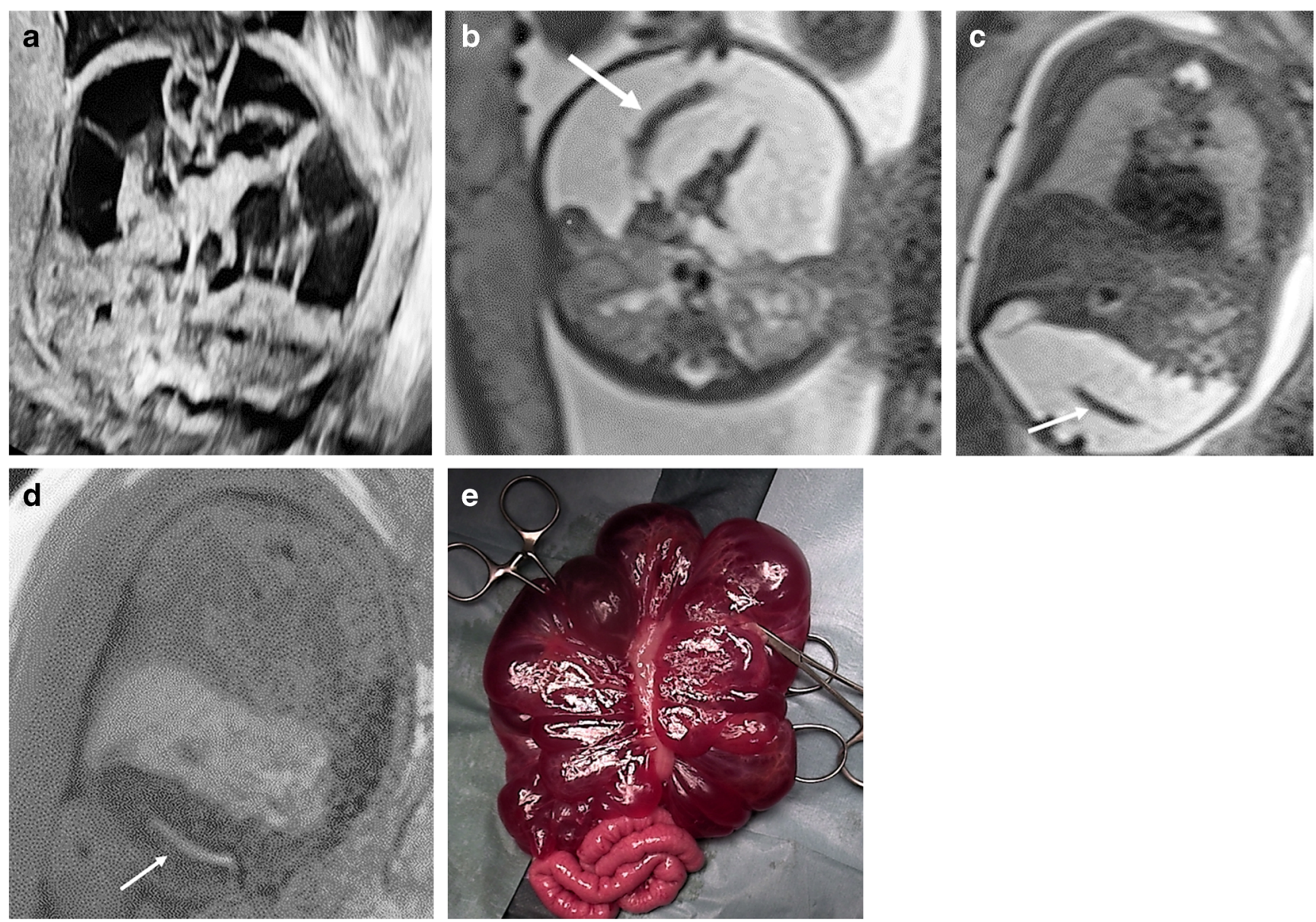

Fig. 2 A male fetus at 35 weeks' gestation with extensive mesenteric malformation and postnatal surgery at 4 months old (N18, Table 3). ad Axial US (a) and T2-weighted MR (b) images (repetition time [TR]/ echo time [TE] 1,200/90 ms) at the same level and coronal T2- (c) (TR/ TE 1,200/90 ms) and T1-weighted (d) (TR/TE 3.29/1.29 ms) MR images show the extensive mesenteric macrocystic lymphatic malformation with homogenous liquid echogenicity and signal intensity and multiple internal septations. The lesion displaces the fetal intraperitoneal organs but shows no significant complication. The fetal colon, surrounded by the lymphatic malformation, is easily identifiable by its meconium filling, hypointense on T2- and hyperintense on T1-weighted images (arrows). The lesion was correctly identified only at MRI. e Postnatal image during the surgical procedure shows the voluminous lesion surrounding the colon method for diagnosing prenatal pathology. However, data concerning its real contribution for congenital vascular anomalies remain limited [12-15].

\section{Congenital lymphatic malformations}

Lymphatic malformation is the most often detected vascular malformation in the fetus and the most widely described in the literature $[4,15-18]$. The reported incidence is $1: 2,000-6,000$ cases in live births, with a slight male predominance [16]. They can occur in any location, but are much more often located in the neck $(75 \%)$ and the axilla $(20 \%)$ than in the abdomen (2\%), limbs (2\%) and mediastinum (1\%) $[4,15$, 17]. They are usually classified as microcystic (cysts $<2 \mathrm{~cm}$ in size), macrocystic $(>2 \mathrm{~cm})$ and mixed lesions. Macrocystic lesions are usually more voluminous and therefore easier to detect in utero than microcystic ones [17]. At US, these malformations appear as multiseptated cystic lesions, lacking solid components, vascularity and calcifications. However, a recent review has described a greater imaging variability than previously reported, including mixed cystic/solid lesions and occasional calcifications [17].

Our series evaluated 20 confirmed lymphatic malformations and obtained a high accuracy in the detection and correct identification of these lesions on both prenatal US and MRI, $80 \%$ and $85 \%$, respectively. All our cervicofacial and axillary located malformations were correctly identified on both methods and compared to previously published series $[4,15,17]$ we had a higher percentage of rare anatomical locations, including the thorax ( 2 cases; $10 \%)$ and the abdomen and/or pelvis ( 3 cases; $15 \%$ ). We observed a significant discordance between the prenatal suggested diagnosis and the final diagnosis in these uncommon locations. US identified only one of two thoracic and none of three abdominal 
Table 5 Additional information provided by late pregnancy MRI exams in patients with lymphatic malformations

\begin{tabular}{|c|c|c|c|c|}
\hline $\mathrm{N}$ & Location & Time of MRI (weeks of pregnancy) & Additional information at $2 \mathrm{nd}$ MRI & Management \\
\hline 3 & Left face + neck & $32 / 35$ & $\begin{array}{l}\text { - Major volume augmentation without hemorrhage } \\
\text { - Oropharynx distortion } \\
\text { - Increasing tracheal compression }\end{array}$ & $\begin{array}{l}\text { - EXIT procedure } \\
\text { - Emergency cesarean at } 36 \\
\text { weeks of pregnancy }\end{array}$ \\
\hline 5 & Face + neck & $26 / 34$ & $\begin{array}{l}\text { - No significant tracheal displacement } \\
\text { - No hemorrhage }\end{array}$ & - Normal birth \\
\hline 11 & Right neck + thorax & $25 / 36$ & - Major volume augmentation due to hemorrhage & $\begin{array}{l}\text { - No need of EXIT } \\
\text { - Elective cesarean at } 37 \\
\quad \text { weeks of pregnancy }\end{array}$ \\
\hline & & & $\begin{array}{l}\text { - Slight lateral tracheal displacement } \\
\text { - Extreme arm abduction }\end{array}$ & - Immediate intubation \\
\hline 12 & Left neck + thorax & $22 / 34$ & $\begin{array}{l}\text { - Mediastinal extension } \\
\text { - Slight tracheal displacement }\end{array}$ & $\begin{array}{l}\text { - No need of EXIT } \\
\text { - Elective cesarean at } 39 \\
\text { weeks of pregnancy }\end{array}$ \\
\hline & & & - No hemorrhage & - Immediate intubation \\
\hline 18 & Abdomen intraperitoneal & $26 / 35$ & $\begin{array}{l}\text { - Major volume augmentation } \\
\text { without hemorrhage } \\
\text { - Increasing abdominal distention } \\
\text { - No intestinal obstruction }\end{array}$ & $\begin{array}{l}\text { - Elective cesarean at } 38 \\
\text { weeks of pregnancy } \\
\text { - Surgical excision at } 2 \\
\text { months of age }\end{array}$ \\
\hline
\end{tabular}

EXIT ex utero intrapartum treatment, $N$ patient number

malformations, whereas MRI identified one thoracic and one abdominal lesion. For the two remaining unidentified cases of abdominal lesions, both methods suggested the teratoma, probably influenced by the presacral location of one of these lesions, typical for this common fetal tumor (Fig. 1).

Atypical imaging findings were the second explanation for the discordance between the prenatal and the final diagnosis. As previously reported, the differentiation between a lymphatic malformation and a fetal teratoma can be extremely difficult in the presence of calcifications, hemorrhage or of mixed cystic and solid lesions [17] Indeed, one of the misinterpreted mesenteric lymphatic malformations showed an extensive hemorrhage with marked heterogenicity in utero.

\section{Additional information provided by MRI}

MRI is less affected than US by fetal position, oligohydramnios, maternal obesity and fetal bone superposition and can more precisely determine the extent of a lesion and its relationship to the adjacent structures $[16,18,19]$. In our series, the information provided by complementary MRI improved the echographic suggested diagnosis and changed the management of $2(10 \%)$ patients. In a thoracic lymphatic malformation, MRI showed an extension toward the mediastinum and into the lungs in addition to voluminous pleural effusions already detected on US, which led to a pregnancy continuation with only comfort care at birth. In a second case, MRI correctly identified a voluminous mesenteric lymphatic malformation and excluded relevant complications, which led to the decision to continue the pregnancy (Fig. 2).

\section{MRI studies performed late in pregnancy}

In cervically located lymphatic malformations, MRI can document the extension toward the mediastinum and evaluate the proximity to the brachial plexus $[16,18]$ or the degree of compression of the airways $[11,18,19]$, information difficult to obtain by US alone [13]. A second MRI exam at advanced pregnancy performed in five lymphatic malformations located in a sensitive anatomical location provided accurate anatomical information and enabled decisions about the type of delivery (Table 5), including the successful performance of an EXIT procedure in a fetus showing increasing tracheal compression (Fig. 3).

\section{Congenital hemangiomas}

Congenital hemangiomas are already fully developed at birth [20]. RICH and NICH types have an almost equal gender distribution, are usually solitary lesions and have a predilection for the skin, mostly in the head or limbs. However, the reported imaging findings of these lesions are still limited, 

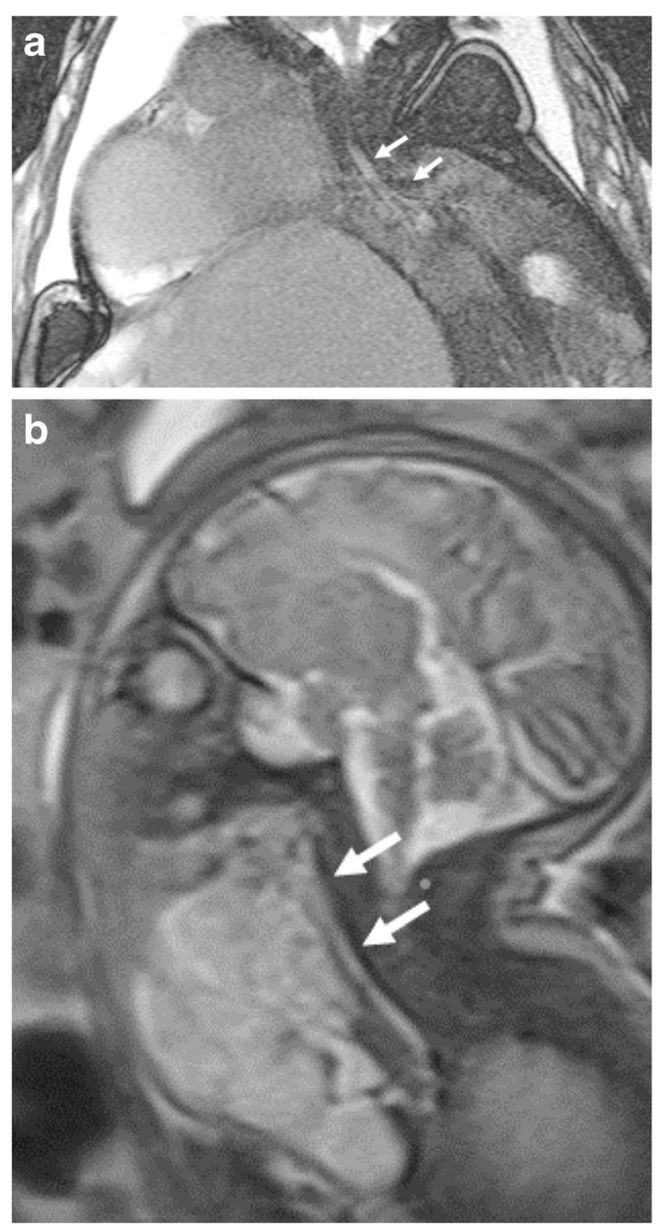

Fig. 3 Cervical lymphatic malformations in a female fetus. a A coronal T2-weighted MR image (repetition time [TR]/echo time [TE] 6.38/ $3.19 \mathrm{~ms}$ ) at 36 weeks' gestation (N11, Tables 3 and 5) shows the voluminous macrocystic lymphatic malformation with heterogeneous signal intensity after intralesional hemorrhage. The cervical mass extends into the axilla and the thoracic and abdominal wall without invading the fetal organs. Note the normal diameter of the trachea and the main bronchi (arrows) and the huge abduction of the fetal arm. $\mathbf{b}$ In opposite, the midline sagittal T2-weighted image (TR/TE 1,200/89 ms) at 35 weeks' gestation (N3, Tables 3 and 5) shows the anterior cervical lymphatic micro- and macrocystic malformation, extending into the submental space without invading the tongue. Note the resulting compression of the larynx and the trachea (arrows). An ex utero intrapartum treatment was performed at birth

with descriptions of isolated cases, concerning mainly RICHtype lesions in typical locations [5, 12, 21].

Hemangiomas are the most frequently detected liver tumors in fetuses and neonates, concerning more than $60 \%$ of all congenital hepatic lesions [22]. They are often heterogeneous on US and may contain identifiable calcifications. On MRI, they also are frequently heterogeneous, with foci of hyperintensity on T1-weighted images and hypointensity on T2-weighted images and intratumoral flow void that represents tubular vascular structures. In a series of 16 children, Franchi-Abella et al. [23] reported well-defined, heterogeneous, hypoechoic lesions compared to the normal liver on US with intratumoral abnormal vessels and enlarged hepatic arteries and/or veins. On MRI, they showed a high signal intensity on T2-weighted images and a low signal intensity on T1-weighted images when compared to the normal liver, with intralesional signal flow voids. Jiao-Ling et al. [24] described well-defined masses on US exams in a series of six congenital hepatic hemangiomas that were mostly hypoechogenic compared to the normal liver with significant rates of heterogeneity, necrosis, cystic cavities and calcifications. Color Doppler showed enlarged hepatic arteries and tortuous, dilated veins. The lesions were hypointense on T1weighted MR images and hyperintense on T2-weighted images compared to the normal liver.

In our series, the sensitivity of prenatal US and MRI for congenital hemangiomas was only $25 \%$, and showed no difference between both prenatal methods. Only one of three hepatic hemangiomas was identified in utero. (Table 4). Strangely, the three hepatic tumors were exophytic, growing from the edge of the left lobe. Two of them had almost identical imaging findings and were hypoechogenic, heterogeneous on US and mostly hyperintense on T2-weighted MR images compared to the normal liver with clearly visible flow voids and an extremely enlarged left hepatic vein (Fig. 4). These two lesions were classified as RICH tumors according to the postnatal evolution. In contrast, the third hemangioma was a rapidly growing, heterogeneous lesion with extensive avascular areas on US Doppler and no intratumoral flow voids identifiable on MRI. Autopsy after emergency cesarean and death at birth revealed a huge hemangioma with extensive areas of hemorrhage, thrombosis and necrosis (Fig. 5).

\section{Additional information provided by MRI}

Complementary MRI influenced the management in a rapidly growing hepatic congenital hemangioma while revealing significant bilateral pulmonary hypoplasia. Emergency cesarean was performed, but the patient died at birth from combined cardiorespiratory insufficiency.

Franchi-Abella et al. [23] suggested that fetal US should remain the standard diagnostic method for hepatic hemangiomas and proposed that MRI should only be performed when the diagnosis is unclear or the lesion is not well-delimited. In opposite, Jiao-Ling et al. [24] and our own results show that voluminous hepatic hemangiomas often have a great imaging variability that could explain the low rates of echographic diagnosis in utero. Although our prenatal rate remained low after MRI, we still believe these exams helped evaluate the effects of the lesions on the fetus.

This article increases the limited available information regarding the prenatal imaging findings of the most frequently 

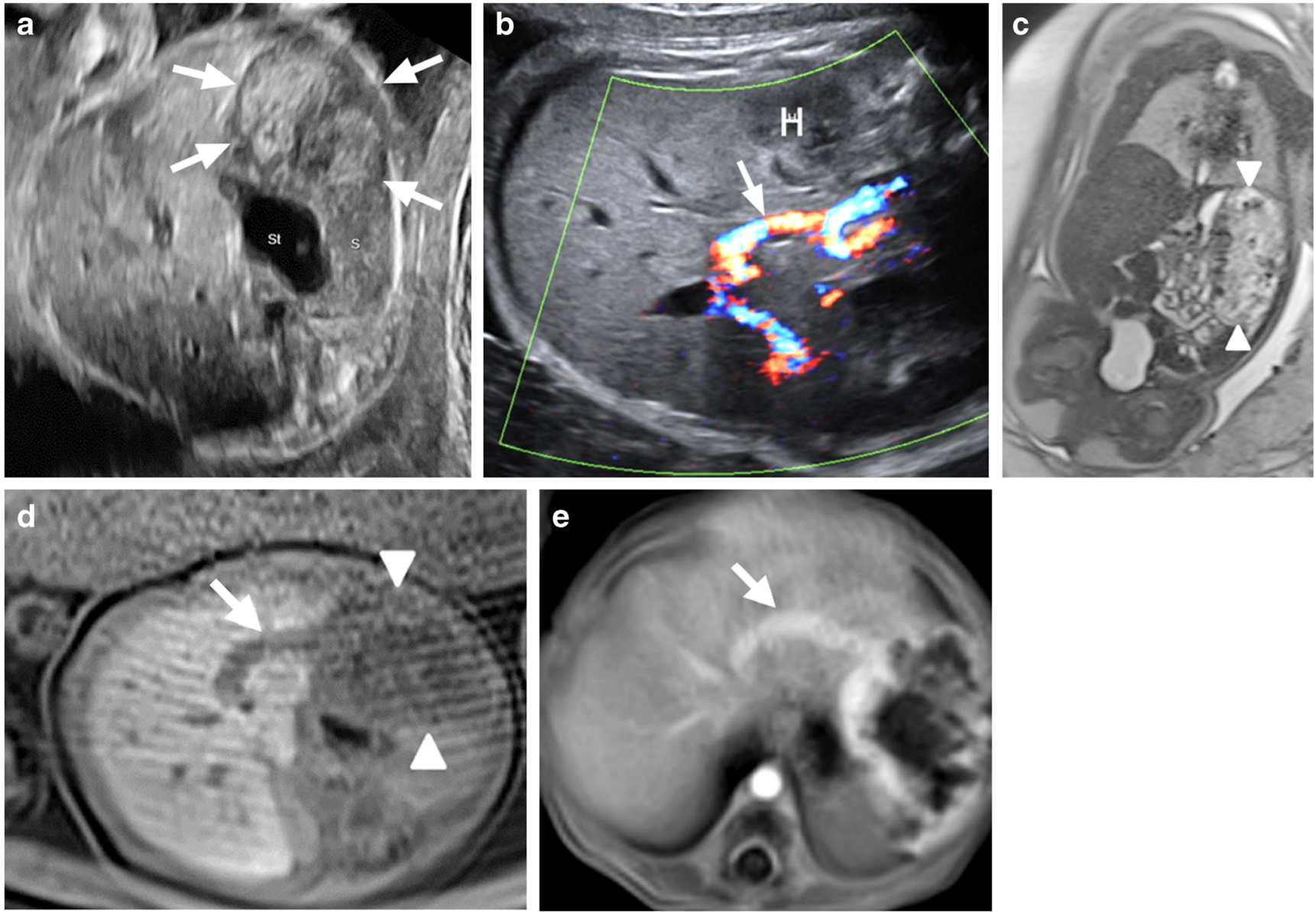

Fig. 4 A male fetus at 33 weeks' gestation (N22) with hepatic hemangioma and postnatal images at 4 days old. a Transverse US image shows a voluminous, solid appearing, heterogeneous left hepatic mass. Arrows indicate the borders of the lesion. $S$ spleen, $S t$ stomach. b-d Axial Doppler US image (b), coronal T2-weighted (c) (repetition time [TR]/echo time [TE] $1,000 / 92 \mathrm{~ms}$ ) image at the same level as well as axial T2-weighted (d) MR image (TR/TE 6.60/3.30 ms) show the inhomogeneous, exophytic growing

lesion arising from the left hepatic lobe ( $H$ in $\mathbf{b}$, arrowheads in $\mathbf{c}, \mathbf{d})$. Note the numerous tubular forming flow voids indicating vascular structures (arrow in d) and the extreme enlarged left hepatic vein (arrow in b). e Postnatal T1weighted MR image (TR/TE 3.61/1.61 ms) after contrast shows the marked early peripheral enhancement of the lesion at the arterial phase and confirms the enlarged left hepatic vein (arrow). After significant regression, the tumor was resected when the boy was 18 months old
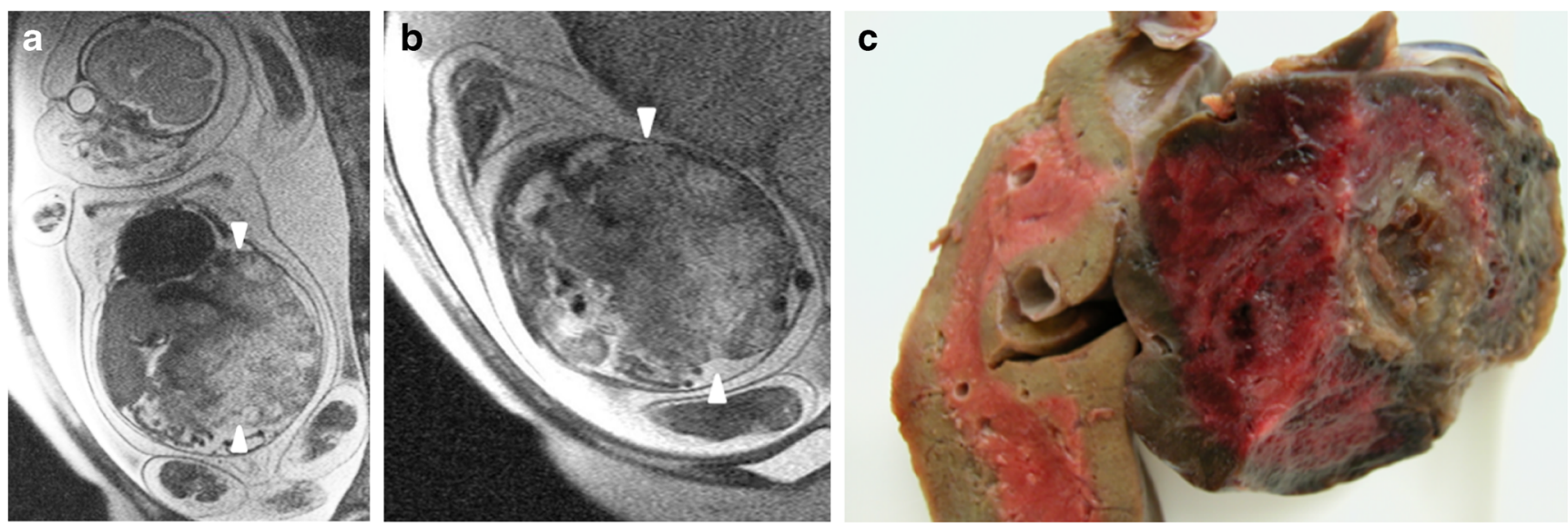

Fig. 5 A male fetus at 27 weeks' gestation with hepatic hemangioma (N23). a-b Coronal (a) and left sagittal (b) T2-weighted MR images (repetition time/echo time 1,530/86 ms) show a huge, heterogeneous left abdominal mass arising from the left hepatic lobe. Note the absence of tubular intralesional structures. Arrowheads show the borders of the lesion. The tumor causes bilateral elevation of the hemidiaphragms and

severe secondary lung hypoplasia, fetal cardiomegaly and marked hydrops fetalis. The patient died at birth after an emergency cesarean at the 27th week of pregnancy. c Macroscopic view of the fetal liver confirms the hepatic origin of this tumor and reveals central cavities and extensive necrosis 
detected congenital vascular anomalies and reveals the main difficulties in their prenatal diagnosis. However, it has some limitations, including its retrospective character and a reduced number of patients despite the participation of three university hospitals. Therefore, these results should be confirmed by more extensive and prospective studies.

\section{Conclusion}

Macrocystic lymphatic malformations and rapidly involuting congenital hemangioma are the most commonly detected congenital vascular anomalies in utero. A rare anatomical location and atypical imaging findings, such as hemorrhage or necrosis, complicate their prenatal diagnosis. The main role of complementary MRI is probably not to improve the diagnosis but to provide high-defined anatomical data to guide the management and anticipate complications at birth.

Acknowledgements Open Access funding provided by Université de Lausanne.

\section{Declarations}

\section{Conflicts of interest None}

Open Access This article is licensed under a Creative Commons Attribution 4.0 International License, which permits use, sharing, adaptation, distribution and reproduction in any medium or format, as long as you give appropriate credit to the original author(s) and the source, provide a link to the Creative Commons licence, and indicate if changes were made. The images or other third party material in this article are included in the article's Creative Commons licence, unless indicated otherwise in a credit line to the material. If material is not included in the article's Creative Commons licence and your intended use is not permitted by statutory regulation or exceeds the permitted use, you will need to obtain permission directly from the copyright holder. To view a copy of this licence, visit http://creativecommons.org/licenses/by/4.0/.

\section{References}

1. Merrow AC, Gupta A, Patel MN, Adams DM (2016) 2014 revised classification of vascular lesions from the International Society for the Study of Vascular Anomalies: radiologic-pathologic update. Radiographics 36:1494-1516

2. Dubois J, Alison M (2010) Vascular anomalies: what a radiologist needs to know. Pediatr Radiol 40:895-905

3. Calvo-Garcia MA, Kline-Fath BM, Adams DM et al (2015) Imaging evaluation of fetal vascular anomalies. Pediatr Radiol 45:1218-1229

4. Wassef M, Vanwijck R, Clapuyt P et al (2006) Vascular tumours and malformations, classification, pathology and imaging. Ann Chir Plast Esthet 51:263-281

5. Chen C-P, Chen C-Y, Chang T-Y et al (2016) Prenatal imaging findings of a rapidly involuting congenital hemangioma (RICH) over right flank in a fetus with a favorable outcome. Taiwan $\mathrm{J}$ Obstet Gynecol 55:745-747

6. Nasseri E, Piram M, McCuaig CC et al (2014) Partially involuting congenital hemangiomas: a report of 8 cases and review of the literature. J Am Acad Dermatol 70:75-79

7. Manganaro L, Antonelli A, Bernardo S et al (2018) Highlights on MRI of the fetal body. Radiol Med 123:271-285

8. Patenaude Y, Pugash D, Lim K et al (2014) The use of magnetic resonance imaging in the obstetric patient. J Obstet Gynaecol Can 36:349-363

9. Flors L, Leiva-Salinas C, Maged IM et al (2011) MR imaging of softtissue vascular malformations: diagnosis, classification, and therapy follow-up. Radiographics 31:1321-1340

10. International Society for the Study of Vascular Anomalies (2018) Classification of vascular anomalies. issva.org/classification. Accessed 28 May 2019

11. Shamshirsaz AA, Stewart KA, Erfani H et al (2019) Cervical lymphatic malformations: prenatal characteristics and ex utero intrapartum treatment. Prenat Diagn 39:287-292

12. Fadell MF 2nd, Jones BV, Adams DM (2011) Prenatal diagnosis and postnatal follow-up of rapidly involuting congenital hemangioma (RICH). Pediatr Radiol 41:1057-1060

13. Olive A, Moldenhauer JS, Laje P et al (2015) Axillary lymphatic malformations: prenatal evaluation and postnatal outcomes. J Pediatr Surg 50:1711-1715

14. Leroy A, Garabedian C, Fourquet $\mathrm{T}$ et al (2016) Iconographic imaging (ultrasound/MRI) in prenatal evaluation of cervical cystic lymphatic malformations. Gynecol Obstet Fertil 44:269-273

15. Chen Y-N, Chen C-P, Lin C-J, Chen S-W (2017) Prenatal ultrasound evaluation and outcome of pregnancy with fetal cystic hygromas and lymphangiomas. J Med Ultrasound 25:12-15

16. Oliveira C, Sacher P, Meuli M (2010) Management of prenatally diagnosed abdominal lymphatic malformations. Eur J Pediatr Surg 20:302-306

17. Oliver ER, Coleman BG, DeBari SE et al (2017) Fetal lymphatic malformations: more variable than we think? J Ultrasound Med 36: $1051-1058$

18. Koelblinger C, Herold C, Nemec S et al (2013) Fetal magnetic resonance imaging of lymphangiomas. J Perinat Med 41:437-443

19. Paula Pinho Matos A, Teixeira Castro P, de Barros DL et al (2018) Prenatal diagnosis of cervical masses by magnetic resonance imaging and 3D virtual models: perinatal and long-term follow-up outcomes. J Matern Fetal Neonatal Med 33:2181-2189

20. Gorincour G, Kokta V, Rypens F et al (2005) Imaging characteristics of two subtypes of congenital hemangiomas: rapidly involuting congenital hemangiomas and non-involuting congenital hemangiomas. Pediatr Radiol 35:1178-1185

21. Richard F, Garel C, Cynober E et al (2009) Prenatal diagnosis of a rapidly involuting congenital hemangioma (RICH) of the skull. Prenat Diagn 29:533-535

22. Isaacs H Jr (2007) Fetal and neonatal hepatic tumors. J Pediatr Surg 42:1797-1803

23. Franchi-Abella S, Gorincour G, Avni F et al (2012) Hepatic haemangioma-prenatal imaging findings, complications and perinatal outcome in a case series. Pediatr Radiol 42:298-307

24. Jiao-Ling L, Xiu-Ping G, Kun-Shan C et al (2018) Huge fetal hepatic hemangioma: prenatal diagnosis on ultrasound and prognosis. BMC Pregnancy Childbirth 18:2

Publisher's note Springer Nature remains neutral with regard to jurisdictional claims in published maps and institutional affiliations. 